Claudia Repossi Côco ${ }^{1}$

Erica Marvila Garcia ${ }^{2}$

Katrini Guidolini Martinelli ${ }^{1}$

Marcelle Lemos Leal ${ }^{1}$

Barbara Almeida Soares Dias ${ }^{3}$

Lorrayne Belotti ${ }^{2}$

\section{Incidence of tuberculosis in Espírito Santo state from 2005 to 2016}

\title{
A incidência de tuberculose no estado do Espírito Santo: análise do período de 2005 a 2016
}

ABSTRACT | Introduction:

Tuberculosis (TB) is one of the major public bealth issues in the world. According to the World Health Organization (WHO), 10.4 million people became ill and 1.5 million people died due to TB in 2015. Objective:

Analyzing tuberculosis incidence trend and featuring the epidemiological and clinical profile of cases recorded in Espirito Santo State from 2006 to 2015. Methods: Time-trend ecological study whose information was collected in the Information System of Reportable Diseases (SINAN), as well as provided by the Brazilian Geography and Statistics Institute (IBGE) -

DATASUS. Tuberculosis incidence rate was calculated and polynomial regression models were estimated to verify the trend of tuberculosis cases.

Trend was significant when the $p$ value of the estimated model was $p<0.05$. Results: In total, 14,187 tuberculosis cases were reported in Espirito Santo State from 2005 to 2016. The second order model $(p<0.001)$ was the one best representing tuberculosis incidence-trend behavior; it presented coefficient of incidence increase from 2006 to 2009 and to constant decrease from $2010 \mathrm{on}$. The metropolitan region recorded the bighest incidence of TB. Men in the age group

20-39 years, brown, with low schooling and living in a urban areas prevailed in the sample.

Based on clinical data, most tuberculosis cases were diagnosed through bacilloscopy; patients presented the pulmonary form of the disease and did not show TB-related comorbidities. Patients were cured in most cases. Conclusion: Identifying these features can belp bealth services with follow-ups provided to this population, which is highly vulnerable to the berein addressed disease, as well as with the development of health promotion actions and active case search in local communities.

Keywords | Tuberculosis; Health profile; Time-series studies.
RESUMO| Introdução: A tuberculose (TB) é um dos principais problemas de saúde pública. Segundo a Organização Mundial de Saúde (OMS), 10,4 milhões de pessoas adoeceram e 1,5 milhões de pessoas morreram por TB em 2015. Objetivo: Analisar a tendência de incidência de tuberculose e caracterizar o perfil epidemiológico e clínico dos casos no Espírito Santo, de 2006 a 2015. Métodos: Estudo ecológico de série temporal, com informações obtidas do Sistema de Informações de Agravos de Notificação (SINAN) e do Instituto Brasileiro de Geografia e Estatística (IBGE) - DATASUS. Calculou-se a taxa de incidência da tuberculose e foram estimados modelos de regressão polinomial para verificar a sua tendência. Considerou-se tendência significativa aquela cujo modelo estimado obteve $\mathrm{p}<0,05$. Resultados: Foram notificados 14.187 casos de TB no Espírito Santo. O modelo que melhor representa o comportamento da tendência da incidência foi o de segunda ordem ( $p<0,001$ ), com acréscimo do coeficiente de 2006 a 2009 e decréscimo constante a partir de 2010. A região metropolitana apresentou a maior incidência. Houve predominância no sexo masculino, na faixa etária de 20 a 59 anos, entre os pardos, entre aqueles com baixa escolaridade e em residentes em área urbana. Dados clínicos revelaram que a maioria foi diagnosticada por baciloscopia, apresentou a forma pulmonar e apresentou-se sem comorbidades relacionadas à TB. A grande maioria dos casos foi encerrada por cura. Conclusão: A identificação dessas características pode auxiliar os serviços de saúde no acompanhamento da população com maior vulnerabilidade para o adoecimento e no desenvolvimento de ações de promoção à saúde e busca ativa de casos nas comunidades.

Palavras-chave| Tuberculose; Perfil epidemiológico; Séries temporais.

${ }^{1}$ Universidade Federal do Espírito Santo. Vitória/ES, Brasil.

${ }^{2}$ Universidade de São Paulo. São Paulo/SP, Brasil.

${ }^{3}$ Fundação Oswaldo Cruz. Rio de Janeiro/RJ, Brasil. 


\section{INTRODUÇÃO|}

A tuberculose (TB) continua sendo mundialmente um dos principais problemas de saúde pública. Segundo a Organização Mundial de Saúde (OMS), 10,4 milhões de pessoas adoeceram e 1,5 milhão de pessoas morreram por TB em 2015². No Brasil, dados do Ministério da Saúde mostram que o coeficiente de mortalidade por tuberculose em 2016 foi de 2,1 mortes por 100mil habitantes ${ }^{2}$.

O Brasil ocupa posição de destaque entre os 22 países que concentram $82 \%$ da carga mundial de TB, e a doença é considerada prioritária na agenda política do Ministério da Saúde, o que exige o desenvolvimento de estratégias para seu controle. Em 2014, durante a Assembleia Mundial de Saúde, foi aprovada a nova estratégia global para enfrentamento da tuberculose, com a visão de um mundo livre da tuberculose até $2035^{1}$.

Seguindo as prerrogativas do Sistema Único de Saúde (SUS), a descentralização para o âmbito da Atenção Primária à Saúde (APS) passa a funcionar como principal porta de entrada para os suspeitos de TB e pacientes com a doença. A Estratégia de Saúde da Família (ESF), estratégia prioritária da atenção primária, apesar de ter aumentado a cobertura em todo o território nacional, ainda precisa qualificar melhor a linha de cuidado da TB, considerando as necessidades dos usuários, bem como as singularidades de cada território ${ }^{3}$.

O Sistema de Informação de Agravos de Notificação (SINAN) é o principal instrumento nacional para coleta e análise dos dados sobre agravos, doenças e eventos de saúde pública, incluindo a TB. As informações por ele geradas auxiliam no planejamento, prevenção, avaliação e controle de doenças, atuando como uma importante ferramenta para a vigilância epidemiológica ${ }^{4}$.

A análise do perfil epidemiológico dessa doença é de extrema importância para o Estado pois, através dos dados analisados, torna-se possível identificar as características da população acometida e suas fragilidades, possibilitando que seja traçado um planejamento de ações vislumbrando a diminuição da incidência de TB no Espírito Santo, de acordo com as prerrogativas do Ministério da Saúde pelo fim da TB como problema de saúde pública.
O objetivo deste estudo, portanto, é analisar a tendência de incidência de tuberculose e caracterizar o perfil epidemiológico e clínico dos casos no Espírito Santo, no período de 2006 a 2015.

\section{MÉTODOS|}

Foi realizado um estudo ecológico de série temporal, de base populacional, baseado nos dados do Sistema de Informação de Agravos de Notificação (SINAN) e do Sistema de Informação do Instituto Brasileiro de Geografia e Estatística (IBGE), integrantes do DATASUS - Ministério da Saúde, relativos ao número de casos de tuberculose e à população residente no Espírito Santo, no período de 2006 a 2015.

Para este estudo, foram utilizadas as seguintes variáveis: raça/cor; escolaridade; sexo; faixa etária; local de infecção; critério de confirmação laboratorial; forma clínica da tuberculose; agravos associados à tuberculose, e situação de encerramento do caso.

Foi realizado o cálculo da incidência de tuberculose segundo região de saúde, sexo e faixa etária. Para tal, dividiu-se o número de casos novos de TB pela população exposta ao risco no mesmo lugar e multiplicou-se por 100.000. Para outras variáveis, calculou-se a frequência absoluta e relativa de cada categoria.

Para análise da tendência da incidência de tuberculose no período de 2006 a 2015, foram feitos diagramas de dispersão para os coeficientes e os anos de estudo, de modo a visualizar a função que poderia expressar a relação entre eles. A partir da relação funcional observada, foram estimados modelos de regressão polinomial.

No processo de modelagem, os coeficientes de incidência de tuberculose foram considerados como variável dependente $(\mathrm{Y})$, e os anos do calendário de estudo como variável independente $(\mathrm{X})$. A transformação da variável "ano" na variável ano-centralizada (ano menos o ponto médio do período de estudo) fez-se necessária porque em modelos de regressão polinomial os termos da equação frequentemente são altamente correlacionados. Assim, expressar a variável independente como um desvio de sua média reduz substancialmente a autocorrelação entre 
eles. Como medida de precisão do modelo, utilizou-se o coeficiente de determinação (r2).

Verificou-se aderência dos dados à distribuição Normal por meio do teste de Kolmogorov-Smirnov, e todas as séries apresentaram distribuição Normal. A análise dos resíduos confirmou a suposição de homocedasticidade do modelo.

Inicialmente, testou-se o modelo de regressão linear simples $(Y=\beta 0+\beta 1 X)$. Em seguida, foram testados os modelos de segundo grau $(\mathrm{Y}=\beta 0+\beta 1 \mathrm{X}+\beta 2 \mathrm{X} 2)$ e terceiro grau $(\mathrm{Y}=\beta 0+\beta 1 \mathrm{X}+\beta 2 \mathrm{X} 2+\beta 3 \mathrm{X} 3)$. Quando dois modelos foram semelhantes do ponto de vista estatístico, optou-se pelo modelo mais simples, ou seja, o de menor ordem. Considerou-se tendência significativa aquela cujo modelo estimado obteve $\mathrm{p}<0,05$.

Os cálculos das taxas de incidência e gráficos com as séries históricas foram elaborados em planilhas do Microsoft Office Excel (Versão 14.0 para Windows 2016), e as análises de tendências foram realizadas no programa STAT $A$, versão 14.1.

Como os dados são de domínio público, não houve necessidade de submeter o projeto de pesquisa à aprovação do Comitê de Ética em Pesquisa da Universidade.

\section{RESULTADOS|}

No período de 2006 a 2015, foram notificados 14.187 casos de tuberculose no Espírito Santo. A raça/cor mais acometida para o período de estudo foi a parda, com percentuais em torno de $50 \%$, seguida pela branca. Mais de $50 \%$ dos acometidos por TB possuíam o ensino fundamental incompleto ou eram analfabetos, e mais de $80 \%$ residiam em área urbana. O percentual de informações ignoradas para raça/cor aumentou ao longo do período, enquanto aquele para escolaridade se manteve elevado, em torno de 15\% (Tabela 1).

Cerca de 70\% dos casos notificados de TB tiveram confirmação laboratorial, e $80 \%$ apresentaram a forma clínica pulmonar. De acordo com a situação de encerramento das notificações, cerca de 75\% foram encerradas por cura, e o percentual de abandono apresentou uma tendência crescente até o ano de 2013, chegando a $11 \%$ (Tabela 2).

Em relação às comorbidades, a maioria passou a ser registrada a partir de 2007. Antes, os percentuais de “ignorado" eram superiores a 85\%. É possível observar que houve um ligeiro aumento dos casos de AIDS e diabetes associados à TB ao longo do período, indo de $6 \%$ para $9 \%$ e de $3,1 \%$ para $7,7 \%$, respectivamente (Tabela 2).

Observou-se que a região metropolitana apresentou a maior incidência em todos os anos de análise quando comparada às outras regiões do Estado, com destaque para o ano de 2008, que apresentou o pico da taxa (50,6/100.000 hab.). No ES, assim como na maioria das regiões, observou-se uma tendência ligeiramente decrescente para o período. Entretanto, houve um aumento na taxa de incidência no ano de 2015 quando comparada àquela de 2014 (Gráfico 1A).

A incidência no sexo masculino foi praticamente o dobro daquela no sexo feminino. Para o período de estudo, a incidência média para os indivíduos do sexo masculino foi de 53/100.000 habitantes. Já para as mulheres, a incidência média foi de 23,3/100.000 habitantes (Gráfico 1B). No que tange à faixa etária, aquela com maior incidência foi a economicamente ativa (20-59 anos), sendo esta a única com tendência a diminuição. As outras mostraram-se estáveis ao longo do período (Gráfico 2).

A Tabela 3 apresenta os resultados da análise das tendências dos coeficientes de incidência por região do Estado. O modelo que melhor representa o comportamento da tendência da incidência de tuberculose no estado de Espírito Santo é o de segunda ordem, com crescimento da taxa nos anos de 2006 a 2009 e decréscimo constante a partir de 2010. Nas regiões Central e Metropolitana, é o modelo linear que melhor explica esse comportamento dos coeficientes. Nessas regiões, houve decréscimo dos coeficientes de $0,82$ ( $\beta 1)$ e 0,89 ( $\beta 1)$, respectivamente. Já as regiões Norte e Sul apresentaram modelo de segunda ordem, ambos com decréscimo do coeficiente a partir de 2010. 


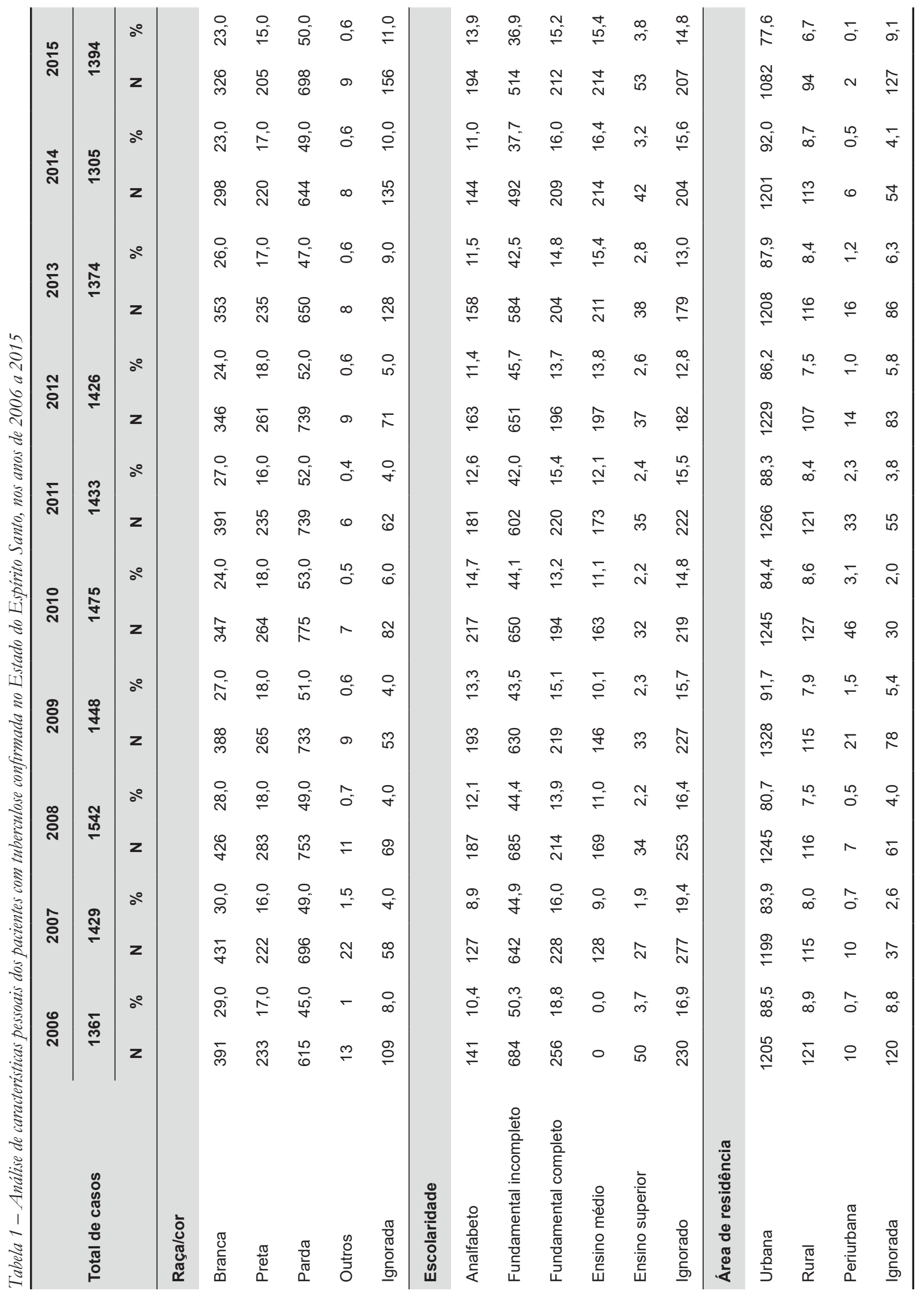




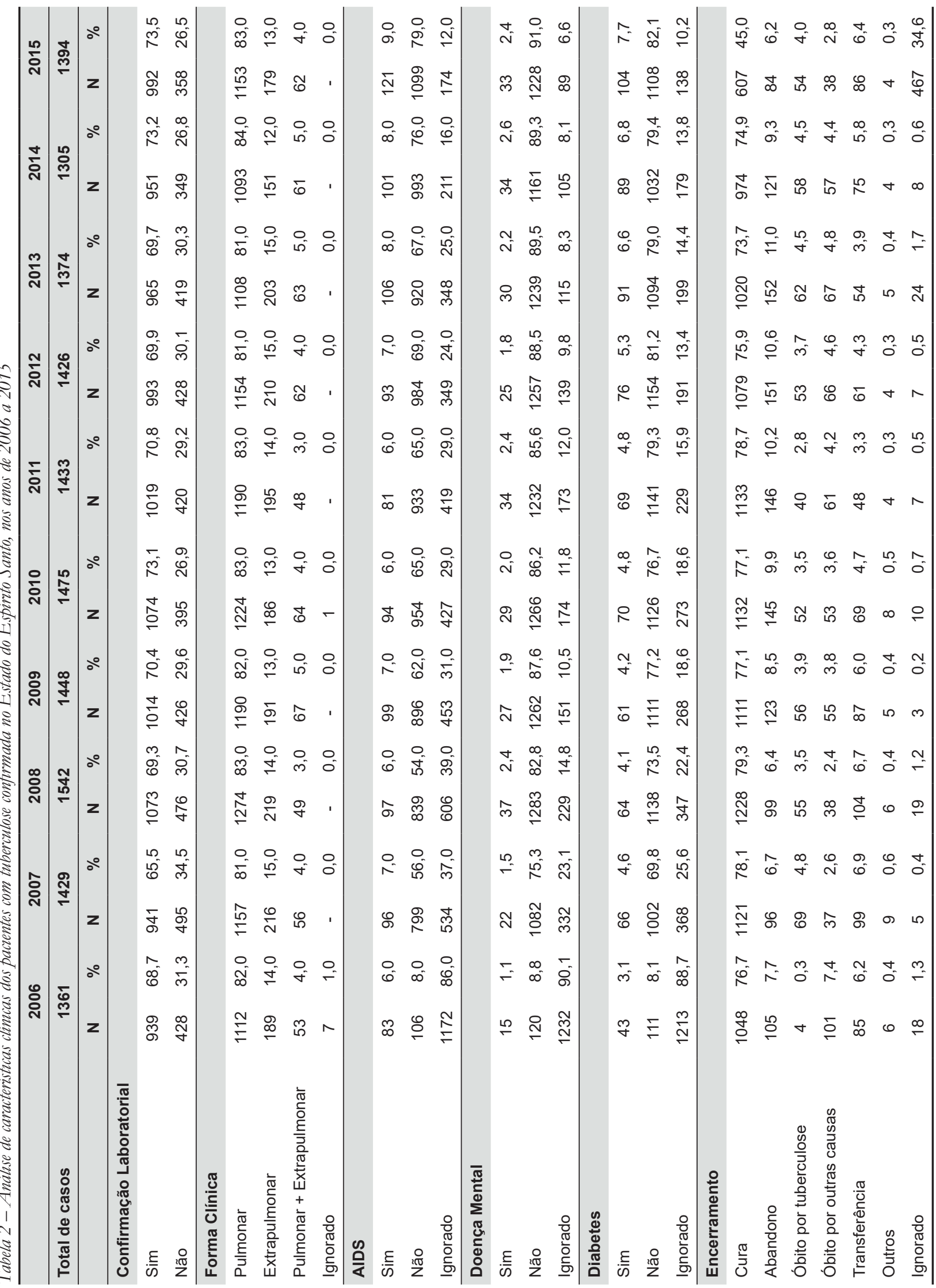


Gráfico 1 - Incidência de tuberculose por 100.000 habitantes no Espírito Santo, segundo região (A) e sexo (B), no Estado do Espírito Santo, no periodo 2006-2015

60,0

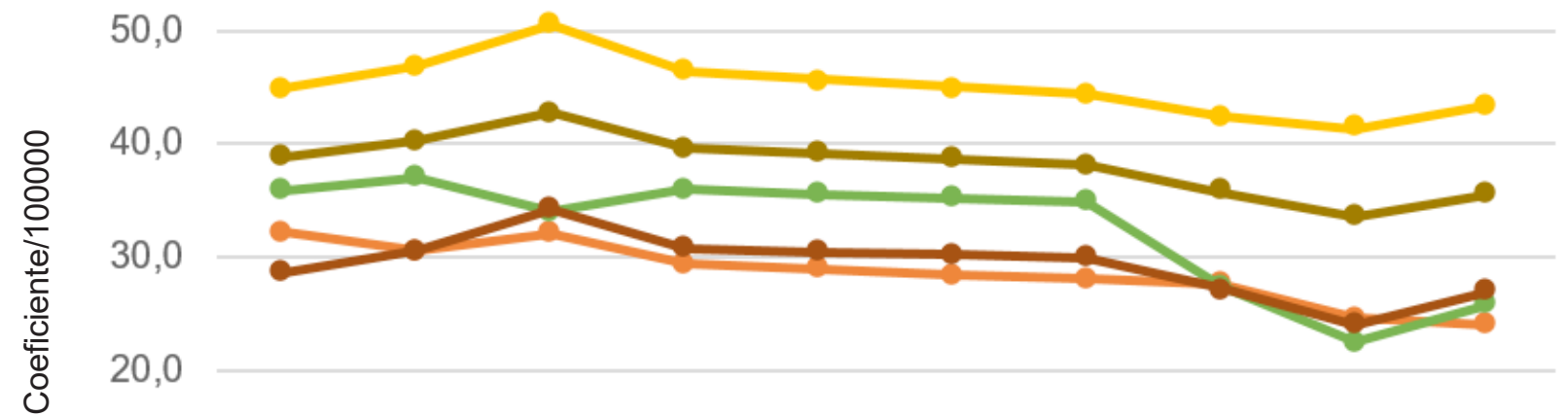

10,0

0,0 $\begin{array}{llllllllll}2006 & 2007 & 2008 & 2009 & 2010 & 2011 & 2012 & 2013 & 2014 & 2015\end{array}$

(A)

$$
\multimap \text { Central } \multimap \text { Metropolitana } \multimap \text { Norte } \multimap \text { Sul } \multimap \text { ES }
$$

70,0

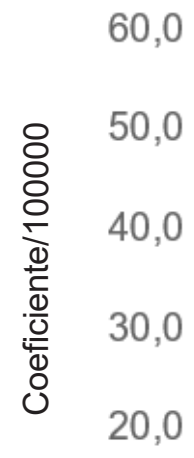
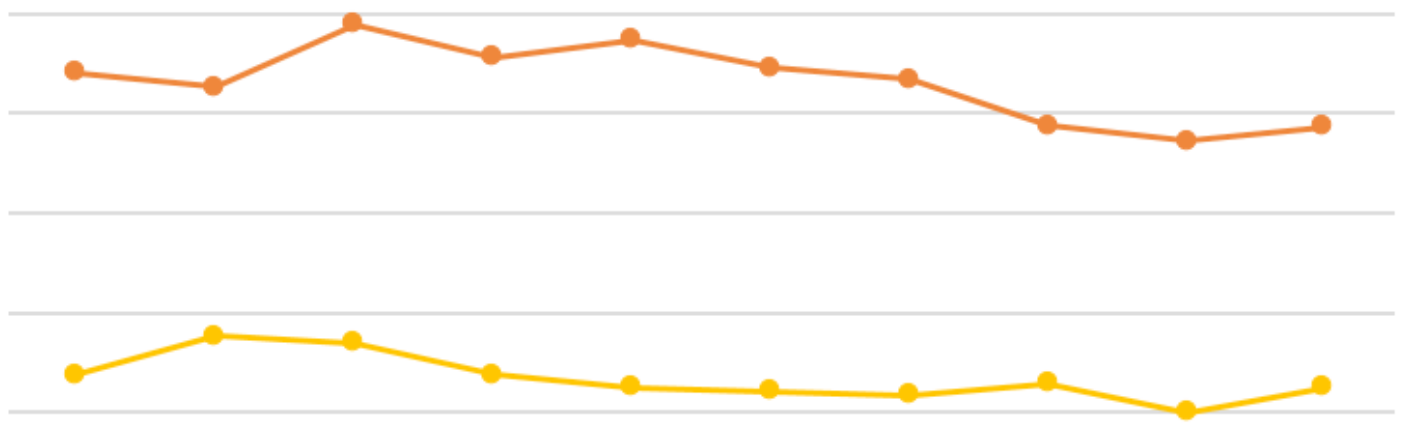

10,0

0,0

$\begin{array}{llllllllll}2006 & 2007 & 2008 & 2009 & 2010 & 2011 & 2012 & 2013 & 2014 & 2015\end{array}$

(B)

$$
\rightarrow \text { Masculino } \longrightarrow \text { Feminino }
$$


Gráfico 2 - Incidência de tuberculose por 100.000 babitantes no Espírito Santo, segundo faixa etária, no período 2006-2015

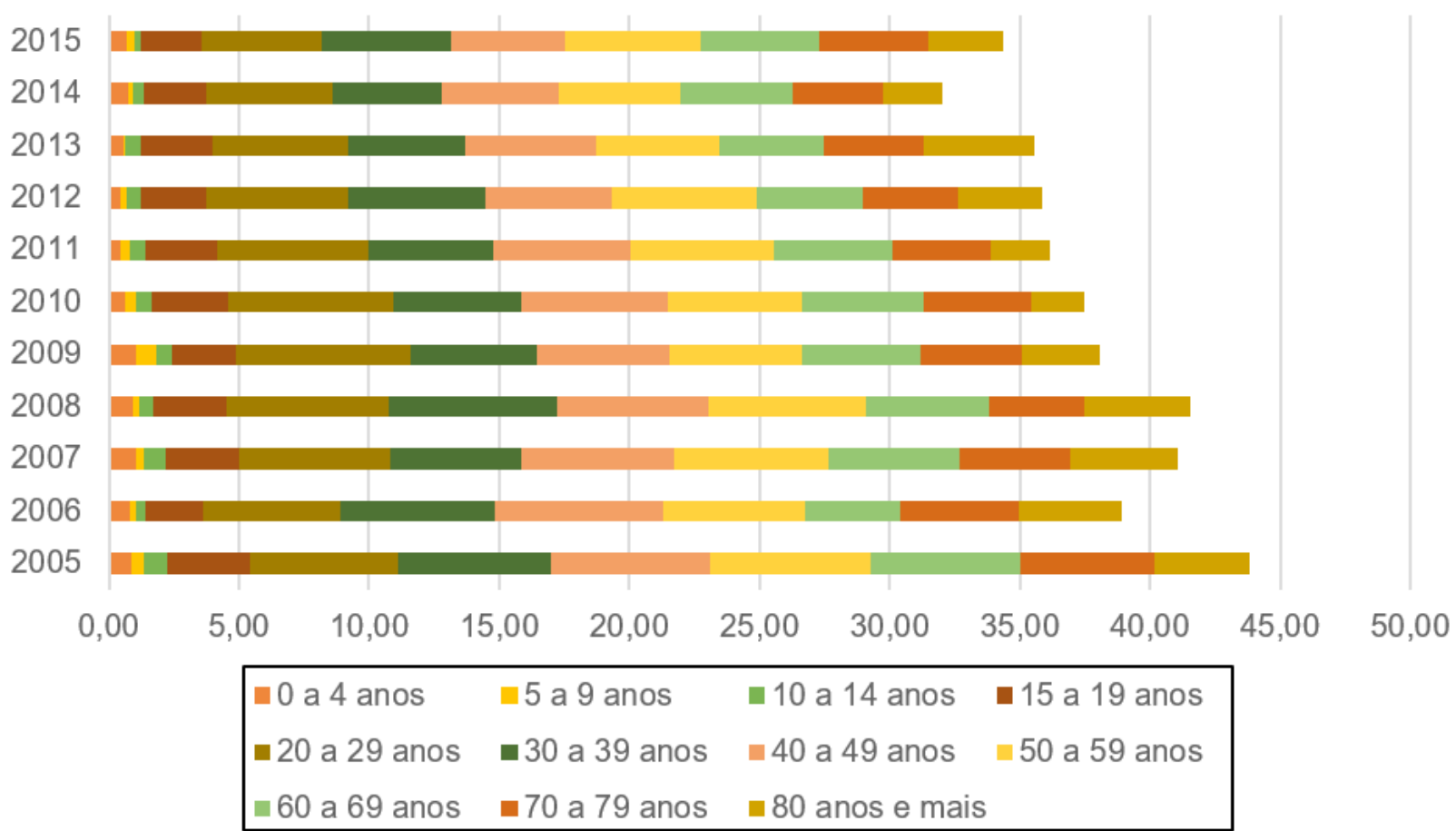

Tabela 3 - Resultados da análise de tendência dos coeficientes de incidência de tuberculose, segundo as regiões do Espírito Santo, no período de 2006 a 2015

\begin{tabular}{|c|c|c|c|c|c|}
\hline Região & Modelo & $\mathbf{R}^{2}(\%)$ & p-valor & Ordem & Tendência \\
\hline Central & $y=28,79-0,82 x$ & 97,76 & $<0,001$ & Linear & Decréscimo \\
\hline Metropolitana & $y=45,61-0,89 x$ & 86,64 & 0,001 & Linear & Decréscimo \\
\hline Norte & $y=35,01-1,41 x-0,39 x^{2}$ & 95,92 & 0,001 & $2^{\circ}$ Ordem & $\begin{array}{l}\text { Acréscimo de } 2006 \text { a } 2009 \text { e } \\
\text { decréscimo a partir de } 2010\end{array}$ \\
\hline Sul & $y=30,59-0,80 x-0,16 x^{2}$ & 96,32 & $<0,001$ & $2^{\circ}$ Ordem & $\begin{array}{l}\text { Acréscimo de } 2006 \text { a } 2009 \text { e } \\
\text { decréscimo a partir de } 2010\end{array}$ \\
\hline Espírito Santo & $y=39,12-0,92 x-0,11 x^{2}$ & 96,65 & $<0,001$ & $2^{\circ}$ Ordem & $\begin{array}{l}\text { Acréscimo de } 2006 \text { a } 2009 \text { e } \\
\text { decréscimo a partir de } 2010\end{array}$ \\
\hline
\end{tabular}

\section{DISCUSSÃO|}

O comportamento da tendência da incidência de tuberculose no estado de Espírito Santo é o de segunda ordem, ou seja, apresentou crescimento da taxa nos anos de 2006 a 2009 e decréscimo nos anos seguintes. A tendência de queda da incidência se repetiu em todas as regiões, porém foi menos evidente na região metropolitana. A TB afetou mais indivíduos pardos, do sexo masculino e com baixa escolaridade. A maioria dos diagnósticos foi realizado por meio da baciloscopia, sendo predominante a forma clínica pulmonar e a cura como principal forma de encerramento.
Observou-se que a incidência da região metropolitana sempre se apresentou com valores superiores em comparação às demais regiões de saúde do ES. Isso está em acordo com o estudo de Fregona et al. ${ }^{5}$ ao relacionar a implantação da Estratégia de Saúde da Família (ESF) no ES com o controle da TB. Semelhantemente, Dias et al. ${ }^{6}$ realizaram estudo de análise espacial da TB infantil no ES e encontraram maior incidência na região metropolitana, em todo o litoral e, principalmente, no município de Vitória.

O fato das incidências anuais serem maiores na região metropolitana pode estar relacionado à presença de 
centros de tratamento de referência, o que pode facilitar o diagnóstico, aumentando a notificação. Outro importante ponto a ser considerado é a aglomeração urbana na região metropolitana, visto que as aglomerações populacionais, aliadas à urbanização acelerada, favorecem altas taxas de desemprego, subemprego, diminuição do nível salarial e pobreza, a que se acrescentam as más condições de habitação e nutrição ${ }^{7}$. Ainda, destaca-se que a TB se distribui heterogeneamente na cidade e apresenta as maiores incidências nos bairros com pior índice de qualidade urbana ${ }^{8}$.

Tendo em vista a alta incidência da tuberculose nos grandes centros urbanos, Maciel e Sales ${ }^{9}$ propõem a construção de um fluxograma de vigilância territorial da tuberculose, abordando a busca de sintomáticos respiratórios por meio da técnica do "ecomapa" para a identificação das relações sociais do indivíduo. Essa abordagem considera a transmissão na comunidade e em seus espaços sociais e não somente o contato intradomiciliar e, portanto, busca organizar e dinamizar os processos de trabalho no âmbito da atenção primária à saúde para o controle da TB.

Houve predominância de tuberculose no sexo masculino. Tal achado coincide com os dados de vários outros estudos da literatura realizados tanto em nível nacional ${ }^{7,10,11}$ quanto em nível estadual ${ }^{6,12,13}$.

Segundo Hino et al. $^{14}$, isso ocorre devido a fatores socioeconômicos e culturais e, também, pela forma como o homem lida com a própria saúde, procurando eventualmente o serviço de saúde. Outros autores relatam que fatores como maior consumo de álcool, tabagismo e a maior procura rotineira aos serviços de saúde pelas mulheres estão entre os fatores responsáveis pela diferença entre os $\operatorname{sexos}^{15,16}$.

Os indivíduos na faixa etária economicamente ativa (20 a 59 anos) foram os mais atingidos, possivelmente por estarem mais expostos aos fatores de risco. Prado et al. ${ }^{12}$, ao estudarem a relação da TB e da AIDS no ES, também encontraram predominância da coinfecção nessa faixa etária. Estudo de Barros et al. ${ }^{17}$ também encontrou predominância na faixa etária mais economicamente ativa ao estudar o perfil epidemiológico da TB extrapulmonar na Paraíba. Esse padrão etário é nacional, causando prejuízo econômico tanto para as famílias afetadas quanto para o mercado de trabalho.
Neste estudo, os indivíduos da raça parda foram os mais afetados, o que está em consonância com outros estudos ${ }^{6,11,18}$. Essas incidências estão geralmente associadas às desigualdades socioeconômicas e às insatisfatórias condições de acesso aos serviços de saúde, o que também justifica o fato de serem as populações indígenas e pretas aquelas mais vulneráveis ao adoecimento por tuberculose, bem como aquelas que encontram maiores dificuldades no acesso aos serviços de diagnóstico e tratamento quando comparadas às outras categorias de raça/cor no país ${ }^{19}$.

Em estudo racial da tuberculose e da paracoccidioidomicose, a dificuldade de classificação devido à grande miscigenação de raças em nosso meio e ao viés cultural existente para a definição de cor/raça se mostrou um fator importante na determinação da categoria dessa variável com base na observação visual do pigmento cutâneo por parte dos responsáveis pelas notificações ${ }^{20}$.

A baixa escolaridade foi predominante na população estudada, seguindo padrão nacional de acometimento de TB e corroborando o resultado de outras pesquisas ${ }^{11,12,18}$.

A baixa escolaridade configura-se como um fator determinante para o aumento da vulnerabilidade social à qual o indivíduo está exposto, aumentando as chances do desenvolvimento da doença, no sentido de que o acesso à informação sobre ela pode estar prejudicado, podendo aumentar o abandono ao tratamento, bem como aumentar o caráter excludente da doença ${ }^{3}$.

As características clínicas da população estudada revelam que $70,21 \%$ realizaram baciloscopia. Os demais foram diagnosticados pela clínica apresentada. Canoa et $\mathrm{al}^{21}$, estudando a TB em pacientes pediátricos, também encontraram 67,6\% com diagnóstico de TB confirmado por baciloscopia. Isso demonstra a importância do exame de escarro para o diagnóstico, dada a sua praticidade e eficácia, apesar de possuir sensibilidade inferior a outros exames, como a cultura do escarro ${ }^{3}$.

A predominância da forma pulmonar entre os casos do estudo coincide com a distribuição estimada para o Brasil pelo Ministério da Saúde (90\%) e com os resultados obtidos em um estudo do perfil epidemiológico de pacientes adultos com tuberculose e AIDS no estado do Espírito Santo ${ }^{12}$. Esse percentual elevado pode ser justificado pelo fato de os pulmões serem órgãos com altas concentrações de oxigênio, tornando-se o local 
preferencial para a instalação do Mycobacterium tuberculosis, bactéria aeróbica estrita ${ }^{22}$.

Neste estudo, cerca de 75\% dos pacientes evoluíram para cura e em cerca de $10 \%$ foi registrado abandono. A OMS preconiza que, para o controle da doença, a meta de cura seja igual ou superior a $85 \%$ e a de abandono seja menor do que 5\% ${ }^{1}$. Qualquer porcentagem, mesmo que mínima, relacionada à situação de encerramento, contrária à cura torna-se um fator extremamente importante, uma vez que pode ocasionar a continuidade da transmissão da doença, e a resistência medicamentosa do bacilo é uma das maiores consequências do tratamento inadequado ${ }^{23}$.

A cura dos pacientes diagnosticados com tuberculose é uma das principais estratégias para redução da morbimortalidade da doença. No ano de 2014, do total de casos novos pulmonares diagnosticados com confirmação laboratorial no país, 75,1\% foram curados e 11,3\% abandonaram o tratamento ${ }^{1}$.

No que diz respeito às comorbidades, a maioria das notificações no ano de 2006 assinalou como ignorada a presença de AIDS, de doença mental, de diabetes e de alcoolismo, mas, a partir de 2007 , as notificações começaram a registrar sim ou não para as categorias do quesito, apontando melhoria na qualidade das informações. Dessa forma, quando houve preenchimento, na maioria dos casos evidenciou-se não haver presença dessas comorbidades.

O não preenchimento de algumas características demonstra a grande fragilidade nos sistemas de informação, visto que a baixa completude dos dados, o não preenchimento correto da ficha de notificação, a subnotificação e a precariedade das informações relacionadas ao encerramento dos casos de TB limitam o real conhecimento do perfil da tuberculose.

\section{CONCLUSÃO|}

O estudo possibilitou identificar tendência decrescente da incidência de tuberculose no estado de Espírito Santo. A maior incidência de TB na região metropolitana apresentouse com predomínio do sexo masculino, na faixa etária de 20 a 29 anos, entre os pardos, naqueles com baixa escolaridade e nos residentes em área urbana. Os dados clínicos revelam que a maioria foi diagnosticada por meio de baciloscopia e apresentou a forma pulmonar, predominando a ausência das comorbidades relacionadas à TB. A grande maioria dos casos foi encerrada por cura.

Esses achados refletem o cenário das desigualdades na dinâmica social e econômica, principal causa da pobreza e da vulnerabilidade social, ambiente favorável para o predomínio das doenças infecciosas como importante causa de morbimortalidade em nosso meio.

A identificação dessas características pode auxiliar os serviços de saúde no acompanhamento da população com maior vulnerabilidade para o adoecimento, além de garantir uma quebra no elo de transmissão da doença e fortalecer o plano nacional pelo fim da tuberculose como problema de saúde pública.

\section{REFERÊNCIAS |}

1. Brasil. Ministério da Saúde. Secretaria de Vigilância em Saúde. Brasil Livre da Tuberculose: Plano nacional pelo fim da tuberculose como problema de saúde pública. Brasília: Ministério da Saúde; 2017.

2. Brasil. Ministério da Saúde. Secretaria de Vigilância em Saúde. Programa nacional de controle da tuberculose. Brasília: Ministério da Saúde; 2018.

3. Brasil. Ministério da Saúde. Secretaria de Vigilância em Saúde. Manual de recomendações para o controle da tuberculose no Brasil. Brasília: Ministério da Saúde; 2011.

4. Moreira CMM, Maciel ELN. Completude dos dados do Programa de Controle da Tuberculose no Sistema de Informação de Agravos de Notificação no Estado do Espírito Santo, Brasil: uma análise do período de 2001 a 2005. J Bras Pneumol. 2008; 34(4):225-9.

5. Fregona G. Contribuição da estratégia saúde da família para o controle da tuberculose no Espírito Santo. Dissertação [Mestrado em Saúde Coletiva]. - Universidade Federal do Espírito Santo; 2007.

6. Dias BAS, Sales CMM, Bertolde AI, Maciel ELN. Análise espacial da tuberculose infantil no Espírito Santo no período de 2001 a 2011. Rev Bras Pesq Saúde. 2014; 16(3):92-8. 
7. Coutinho LASA, Oliveira DS, Souza GF, Fernandes Filho GMC, Saraiva MG. Perfil epidemiológico da tuberculose no município de João Pessoa - PB, entre 2007 - 2010. Rev Bras Ciênc Saúde. 2012; 16(1):29-35.

8. Vieira RCA, Prado TN, Siqueira MG, Dietze R, Maciel ELN. Distribuição espacial dos casos novos de tuberculose em Vitória, Estado do Espírito Santo, no período entre 2000 e 2005. Rev Soc Bras Med Trop. 2008; 41(1):82-6.

9. Maciel ELN, Sales CMM. A vigilância epidemiológica da tuberculose no Brasil: como é possível avançar mais? Epidemiol Serv Saúde. 2016; 25(1):175-8.

10. Freitas WMTM, Santos CC, Silva MM, Rocha GA. Perfil clínico-epidemiológico de pacientes portadores de tuberculose atendidos em uma unidade municipal de saúde de Belém, Estado do Pará, Brasil. Revista Pan-Amaz Saúde. 2016; 7(2):45-50.

11. Mascarenhas MDM, Araújo LM, Gomes KRO. Perfil epidemiológico da tuberculose entre casos notificados no Município de Piripiri, Estado do Piauí, Brasil. Epidemiol Serv Saúde. 2005; 14(1):7-14.

12. Prado TN, Caus AL, Marques M, Maciel EL, Golub JE, Miranda AE. Perfil epidemiológico de pacientes adultos com tuberculose e AIDS no estado do Espírito Santo, Brasil: relacionamento dos bancos de dados de tuberculose e AIDS. J Bras Pneumol. 2011; 37(1):93-9.

13. Gomes T. Tuberculose extrapulmonar: uma abordagem epidemiológica e molecular. Vitória. Dissertação [Mestrado em Doenças Infecciosas] - Universidade Federal do Espírito Santo; 2013.

14. Hino P, Cunha TN, Villa TCS, Santos CB. Perfil dos casos novos de tuberculose notificados em Ribeirão Preto (SP) no período de 2000 a 2006. Ciênc Saúde Coletiva. 2011; 16(Supl. 1):1295-301.

15. Monteiro PC, Gazetta CE. Aspectos epidemiológicos, clínicos e operacionais do controle da tuberculose em um Hospital Escola - 1999 a 2004. Arq Ciênc Saúde. 2007; 14(2):99-106.

16. Pinheiro RS, Viacava F, Travassos C, Brito AS. Gênero, morbidade, acesso e utilização de serviços de saúde no Brasil. Ciênc Saúde Coletiva. 2002; 7(4):687-707.
17. Barros PG, Pinto ML, Silva TC, Silva EL, Figueiredo TMRM. Perfil epidemiológico dos casos de tuberculose extrapulmonar em um município do estado da Paraíba, 2001-2010. Cad Saúde Colet. 2014; 22(4):343-50.

18. Pereira JC, Silva MR, Costa RR, Guimarães MDC, Leite ICG. Perfil e seguimento dos pacientes com tuberculose em município prioritário no Brasil. Rev Saúde Pública. 2015; 49(6):1-12.

19. Viana PVS. Tuberculose no Brasil: uma análise dos dados de notificação, segundo macro-região e raça/cor, para o período 2008-2011. Rio de Janeiro. Dissertação [Mestrado em Epidemiologia em Saúde Pública] - Escola Nacional de Saúde Pública Sergio Arouca; 2014.

20. Quagliato Junior R, Capitani EM, Zambon L, Rezende SM, Balthazar AB, Bertuzzo CS, et al. Estudo racial na tuberculose e paracoccidioidomicose. Rev Bras Med. 2005; 62(7):295-97.

21. Canoa APG, Romaneli MTN, Pereira RM, Tresoldi AT. Tuberculose em pacientes pediátricos: como tem sido feito o diagnóstico? Rev Paul Pediatr. 2017; 35(2):165-70.

22. Castelo Filho A, Kritski AL, Barreto ÂW, Lemos ACM, Ruffino Netto A, Guimarães CA. II consenso brasileiro de tuberculose: diretrizes brasileiras para tuberculose 2004. J Bras Pneumol. 2004; 30(Supl. 1):57-86.

23. Nogueira PA, Abrahão RMCM, Malucelli MIC. Baciloscopia de escarro em pacientes internados nos hospitais de tuberculose do Estado de São Paulo. Rev Bras Epidemiol. 2004; 7(1):54-63.

\section{Correspondência para/Reprint request to:}

\section{Lorrayne Belotti}

Faculdade de Saúde Pública, Universidade de São Paulo, Av. Dr. Arnaldo, 715,

São Paulo/SP, Brasil

CEP: 01246-904

Tel.: (27) 992814531

E-mail: lorraynebelotti@usp.br

Submetido em: 15/11/2018

Aceito em: 25/01/2019 